bioRxiv preprint doi: https://doi.org/10.1101/2021.11.15.468756; this version posted November 19,2021 . The copyright holder for this

preprint (which was not certified by peer review) is the author/funder. All rights reserved. No reuse allowed without permission.

Advanced cancer biomarker studies with cSurvival

\title{
cSurvival: a web resource for biomarker interactions in cancer outcomes
}

Xuanjin Cheng ${ }^{1-3^{*}}$, Yongxing Liu ${ }^{1-3}$, Jiahe Wang ${ }^{1-3}$, Yujie Chen ${ }^{1-3}$, A. Gordon Robertson ${ }^{4}$, Xuekui Zhang $^{5}$, Steven J. M. Jones ${ }^{3-4}$, and Stefan Taubert ${ }^{13^{*}}$

${ }^{1}$ Centre for Molecular Medicine and Therapeutics, The University of British Columbia, Vancouver, British Columbia, Canada, ${ }^{2}$ British Columbia Children's Hospital Research Institute, Vancouver, British Columbia, Canada, ${ }^{3}$ Department of Medical Genetics, The University of British Columbia, Vancouver, British Columbia, Canada, ${ }^{4}$ Canada's Michael Smith Genome Science Centre at BC Cancer, Vancouver, British Columbia, Canada, ${ }^{5}$ Department of Mathematics and Statistics, University of Victoria, Victoria, British Columbia, Canada.

* To whom correspondence should be addressed. Tel: +1-604-875-3860; Fax: +1 604-875-3819;

Email: jcheng@cmmt.ubc.ca, taubert@cmmt.ubc.ca

\section{Key points:}

- We developed cSurvival, an advanced framework using clinical correlations to study biomarker interactions in cancers, with source code and curated datasets freely available for all

- cSurvival includes new algorithms to identify optimal cutoffs for two continuous predictors to stratify patients into risk groups, enabling for the first time joint analysis with two genomic predictors;

- cSurvival allows survival analysis at the gene set (GS) level with comprehensive and up-todate GS libraries

- The cSurvival pipeline integrates clinical outcomes and experimental cancer cell line data to generate synergistic biological insights and to mine for appropriate preclinical cell line tools

- cSurvival is built on a manually curated cancer outcomes database

Keywords: genetic interaction; survival analysis; TCGA; TARGET; DepMap; biomarker 


\section{ABSTRACT}

Survival analysis is a technique to identify prognostic biomarkers and genetic vulnerabilities in cancer studies. Large-scale consortium-based projects have profiled $>11,000$ adult and $>4,000$ paediatric tumor cases with clinical outcomes and multi-omics approaches. This provides a resource for investigating molecular-level cancer etiologies using clinical correlations. Although cancers often arise from multiple genetic vulnerabilities and have deregulated gene sets (GSs), existing survival analysis protocols can report only on individual genes. Additionally, there is no systematic method to connect clinical outcomes with experimental (cell line) data. To address these gaps, we developed cSurvival (https://tau.cmmt.ubc.ca/cSurvival). cSurvival provides a user-adjustable analytical pipeline with a curated, integrated database, and offers three main advances: (a) joint analysis with two genomic predictors to identify interacting biomarkers, including new algorithms to identify optimal cutoffs for two continuous predictors; (b) survival analysis not only at the gene, but also the GS level; and (c) integration of clinical and experimental cell line studies to generate synergistic biological insights. To demonstrate these advances, we report three case studies. We confirmed findings of autophagydependent survival in colorectal cancers and of synergistic negative effects between high expression of SLC7A11 and SLC2A1 on outcomes in several cancers. We further used cSurvival to identify high expression of the Nrf2-antioxidant response element pathway as a main indicator for lung cancer prognosis and for cellular resistance to oxidative stress-inducing drugs. Together, these analyses demonstrate cSurvival's ability to support biomarker prognosis and interaction analysis via gene- and GS-level approaches and to integrate clinical and experimental biomedical studies. 


\section{INTRODUCTION}

Survival analysis, or, more broadly, time-to-event analysis, assesses the statistical association between potential risk factors and the time to an event such as death or disease recurrence [1,2]. In both basic and clinical cancer biology studies, survival analysis is an important technique for identifying prognostic biomarkers and genetic vulnerabilities. Experimentally, it is useful for hypothesis generation and mechanistic inference. Clinically, it may help stratify patients into subgroups with distinct risk profiles and guide therapeutic decision making [3,4].

Since 2006, consortium-based projects, such as The Cancer Genome Atlas (TCGA) and the Therapeutically Applicable Research to Generate Effective Treatments (TARGET), have gathered clinicopathologic data along with multi-omics molecular profiles of more than fifteen thousand adult and paediatric human tumors across diverse cancer types [5-7]. Such large data resources allow exploration into cancers at the molecular level using clinical correlations at an unprecedented scale [6].

Despite the importance of survival analysis and the richness of tumor molecular datasets, we find that currently available tools do not fully exploit the potential of survival analysis. First, existing tools can only analyze one genomic predictor at a time, typically mutation or expression of an individual gene [8-23]. However, cancer often occurs due to multiple (epi-) genomic alterations, and incorporating more than one predictor in survival analysis could identify interactions between such alterations. Such interaction analysis could also be used to screen for synthetic lethality, or to identify compensatory targets for non-targetable drivers [24-27]. This in turn could facilitate the development of combination therapies (e.g. drug cocktails), which have higher efficacy and milder side effects than mono-therapy (aka one-gene-one-drug) approaches [28,29]. Second, existing tools support analysis only at the single-gene level [8-23]; however, molecular dysregulations in cancers may involve gene sets (GSs) [30], in which a collection of genes act in concert. For example, a GS may represent a specific pathway (e.g. transforming growth factor- $\beta$ (TGF- $\beta$ ) -mediated SMAD signaling), biological process (e.g. cell cycle), disease (e.g. hereditary nonpolyposis colorectal cancer), or treatment (e.g. chemotherapy) [31,32]. Given this, analysis of prognostic biomarkers at the GS level rather than at the single-gene level should be informative [33-35]. Third, experimentally derived cancer cell line viability data [36-38] and multi-omics profiling [39] provide valuable in vitro information on genetic dependencies and interactions; however, no existing tool connects clinical data to such experimental studies. This makes it difficult to identify suitable preclinical cell line tools to investigate molecular mechanisms underpinning poor prognosis.

Motivated by the lack of suitable tools to address the above challenges, we built cSurvival (Figure 1). Its major advances are:

(i) Joint analysis with two genomic predictors on a wide range of individual cancer types or combinations of cancer types, including new algorithms to search for optimal cutoffs in combinations of two continuous predictors in order to stratify patients into risk groups. The two predictors can include any combination of a wide range of parameters including gene or GS expression, somatic mutation, microRNA expression, DNA methylation, and protein expression; 
(ii) Survival analysis at the GS level with comprehensive and up-to-date GS libraries from the easy Visualization and Inference Toolbox for Transcriptome Analysis (eVITTA) project [40]; and

(iii) A pipeline to integrate clinical outcomes and experimental cancer cell line data.

We have combined a curated and integrated cancer outcomes database with a refined analytical pipeline and customizable visualizations into the cSurvival webserver so that nonprogrammers can use it. In the work described here, we demonstrate cSurvival's capabilities with three case studies. We not only recapitulated reported cancer biomarkers and their interactions, but also identified genetic regulations consistent with published studies, demonstrating that cSurvival's advanced pipeline facilitates cancer biomarker studies.

\section{MATERIAL AND METHODS}

\section{Data extraction and processing}

TCGA: We extracted curated clinical outcome endpoints data from the TCGA Pan-Cancer Clinical Data Resource (TCGA-CDR) [6] and multi-omics molecular data from [41]. We removed 2,614 lowquality samples (Do_not_use=True) and flagged additional 507 problematic cases based on comments in the merged_sample_quality_annotations.tsv file (https://gdc.cancer.gov/node/977). We removed these 507 cases (https://tau.cmmt.ubc.ca/cSurvival/project data/977/flagged cases.tsv) by default, but a user can choose to include them via the web interface. Next, we used TCGA sample type codes (https://gdc.cancer.gov/resources-tcga-users/tcga-code-tables/sample-type-codes) to extract tumor samples: for solid tumors we extracted primary solid tumor (01) samples; for acute myeloid leukemia (LAML) we extracted primary blood derived tumors (03 and 09); for skin cutaneous melanoma (SKCM) we extracted both primary solid (01) and metastatic tumors (06). Then, from [41], we used: batch-corrected, upper quartile-normalized RSEM data; merged somatic mutation calls from the Multi-Center Mutation Calling in Multiple Cancers (MC3) project [42]; purity- and ploidy-corrected, gene-level, thresholded somatic copy number (CN) data; batch-corrected, reads per million (RPM) data for expressed microRNA (miRNA) mature strands; beta values from Illumina HumanMethylation27 (HM27) and HumanMethylation450 (HM450) arrays; and batch-corrected reverse phase protein array (RPPA) data. For duplicated tumor samples, for gene expression, miRNA expression, DNA methylation, and RPPA data, we calculated the geometric means as the final readouts. We further used the annotations in [43] to map HM27 and HM450 probe IDs with chromosomal coordinates and adjacent genes.

TARGET: We extracted clinical and multi-omics data from the NCI Genomic Data Commons [44] with TCGAbiolinks v2.16.4 [45-47]. As above, we extracted primary tumor samples (01 for solid tumors, 03 and 09 for blood-derived). We used upper quartile normalized Fragments Per Kilobase of transcript per Million mapped reads (UQ-FPKM) data for gene expression analysis, and open-access somatic mutation calls for mutation analysis. We converted Ensembl gene IDs into HUGO symbols and Entrez IDs using org.Hs.eg.db v3.11.4 [48].

DepMap: We extracted cell line annotation, mutation, gene expression, CN, CRISPR-Cas9, RNA interference (RNAi), and drug sensitivity data from DepMap 21Q3 [37-39,49], normalized protein 
expression levels from the CCLE proteomics (TS2) database (accessed on 5/19/2021) [50], and drug information from the Drug Repurposing Hub v3/24/2020 [51].

\section{Calculating GS expression}

After a user selects a cancer type or combinations of cancer types, we first transform the normalized gene expression counts of all samples in the selected cancer type(s) into z-scores. Then, for each sample, we computed the expression of a GS as the average expression z-score of all genes within the GS [34].

\section{Survival analysis}

Censoring: A user chooses a censoring time in days, months (30.4375 days), or years ( 365.25 days) (default: 10 years). For a selected clinical endpoint (e.g. overall survival (OS), progression-free survival (PFS)), if the time-to-event is larger than the defined time, we set censoring status to 0 and time to the defined time; if the time-to-event is smaller than or equal to the defined time, we set censoring status to 1 and time stays unchanged.

Survival analysis: We apply Kaplan-Meier (KM) log-rank tests (default) and Cox proportionalhazards $(\mathrm{PH})$ regression models to assess the association with prognosis, using survival v3.2.11 [52]. In joint analysis with two predictors, we use the KM log-rank test (default) or Cox PH likelihood ratio test to assess the overall significance of any difference between the four subgroup combinations of two predictors (Supplementary Figure 1). In addition, we apply Cox PH regression models to assess how two predictors jointly impact outcomes by calculating the effect sizes (hazard ratios, HRs) and significances of the two predictors and their interaction from the fitted regression model. Alternatively, users select a risk subgroup of interest, then we apply KM log-rank test (default) or Cox PH likelihood ratio test to assess the difference between the selected subgroup and the rest of the cases.

Determining optimal cutoffs for continuous predictors: By default, for analysis with a single continuous predictor (gene expression, miRNA expression, DNA methylation, protein expression, cell line unthresholded $\mathrm{CN}$ ) and joint analysis with combinations of continuous and categorical predictors, we determine optimal cutoffs using the minimum $P$-value (default: KM log-rank) method [53] by testing from the lowest (default 0.2 ) to the highest (default 0.8 ) percentile with a defined step (default 0.1 ). In joint analysis with combinations of two continuous predictors, we determine optimal cutoffs using a median-anchored greedy (default) or an exhaustive search (described below, and in Figure 1). Because multiple tests are conducted in searching for optimal cutoffs, we apply a $P$-value correction method to control for false positive probability (described below).

- Median-anchored greedy search: We construct a 2D grid using percentiles of both predictors (Figure 1). Next, we determine the starting point for greedy search by locating the minimum $P$-value computed from testing each percentile in predictor $B$ against the median percentile in predictor $A$. Then, we test the nearest three unexplored points; if a lower $P$-value is found, we move the search to that newly found minimum $P$-value point and test the nearest unexplored points until no lower $P$-value can be found. We only test percentile combinations giving at least $10 \%$ (default) of total cases in each subgroup or subgroup combination. 
- Exhaustive search: We construct a 2D grid using percentiles of both predictors (Figure 1). Next, we determine the optimal percentile combination by locating the minimum $P$-value computed from testing each percentile in predictor $B$ against each percentile in predictor $A$. As above, we only test percentile combinations giving at least $10 \%$ (default) of total cases in each subgroup or subgroup combination.

Permutation-based multiple testing adjustment for optimally selected cutoffs: We use permutations to correct the multiple testing arising from assessing a sequence of candidate cutoffs with the minimum $P$-value method [54]. Briefly, we randomly permute outcomes values (in TCGA and TARGET, survival days and censoring status; in DepMap, gene perturbation effects or drug sensitivity scores) over the samples, and determine the new optimal cutoff. We repeat this a defined number of times (default: $\mathrm{n}=100$ ) to generate the null distribution of the minimum $P$-values, i.e. the empirical distribution for the minimum $P$-values when there is no association between the biomarkers and the survival outcomes. Then, we calculate an empirically adjusted $P$-value ( $P$.adj) by comparing the observed minimum $P$-value to this empirical null distribution. To speed up the calculation, we use mclapply $\mathrm{v} 4.0 .3$ [55] for parallel processing.

\section{Differential dependency and cell viability analysis}

For two-group comparisons, we use a two-tailed two-sample Wilcoxon test (wilcox.test [55]) to assess the differences in dependency scores (CRISPR-Cas9, RNAi) or cell viabilities (drug sensitivity assays). For comparisons between more than two groups, we use a Kruskal-Wallis rank sum test (kuskal.test [55]) to test the overall significance of any difference between subgroups. For continuous genomic predictors, we determine optimal cutoffs and apply multiple testing adjustment, as described above.

\section{Customizable and interactive visualizations}

We generate survival curves and forest plots with survminer v0.4.9 [56]. We also create interactive visualizations for further analysis with ggplot2 v3.3.5 [57] and plotly v4.9.4.1 (https://plotly.com/) (Figure 1): (a) density and box plots showing distribution of dependency scores (DepMap); (b) line plots showing $P$-values and HRs tracked over percentiles; (c) heatmaps showing $P$-values and HRs searched over percentile combinations; (d) bar plots showing distribution of somatic mutations; (e) scatter plots analyzing correlations between two continuous predictors; and (f) violin plots assessing differences in values of a continuous predictor between two categories (e.g. expression differences of a pathway between mutated vs. nonmutated groups). Each visualization is customizable with its own plotting parameters (e.g. colors, time intervals on the x-axis), and data points of interest are searchable and highlightable in box, scatter, and violin plots.

Correlation analysis in scatter plots: We use Pearson's product-moment correlation (default), Kendall's rank correlation tau, and Spearman's rank correlation rho (cor.test [55]) to measure correlations between two continuous predictors. 
Group mean analysis in violin plots: We use a two-tailed two-sample Wilcoxon test (wilcox.test [55]) to assess the differences in a continuous predictor (e.g. gene expression) between two subcategories of a categorical predictor (e.g. loss-of-function mutations vs. other).

\section{Web interface implementation}

We implement the web interface of cSurvival using Apache (v2.4.29, https://httpd.apache.org ), R (v4.0.3, https://www.r-project.org/), R Shiny (v1.5.0, https://CRAN.R-project.org/package=shiny), and R Shiny Server (v1.5.14.948, https://rstudio.com/products/shiny/download-server/). We use plumber (v1.1.0, https://CRAN.R-project.org/package=plumber) and pm2 (v5.1.1, https://pm2.keymetrics.io/) to host cSurvival's API.

\section{RESULTS}

In cSurvival v1.0.0, we have aggregated the following data. From the TCGA and the TARGET projects, clinical and multi-omics data of 10,973 adult and 4,995 paediatric tumors across 40 cancer types (33 adult, 7 paediatric). From the Dependency Map (DepMap) project [36], genetic perturbation data from 17,393 and 17,309 genes screened via CRISPR-Cas9 and RNAi in 1,032 and 712 cell lines, respectively, as well as cell viability data from 4,686 drug compounds screened in 578 cell lines and multi-omics data of 1,747 cell lines. From the eVITTA project v1.2.13 [40], 120,953 GSs (Figure 2).

We performed three case studies to demonstrate cSurvival's capabilities.

First, we tested cSurvival's analytical pipeline at the level of GSs. We recapitulated the finding that high expression of an autophagy signature (Gene Ontology [GO]: 0010506) is associated with poor overall survival in colorectal cancers (colon adenocarcinoma (TCGA-COAD) and rectum adenocarcinoma (TCGA-READ)) (Figure 3A-B, Supplementary Figure 2A) [34].

Second, we performed joint analysis with gene expression data of solute carrier family 7 member 11 (SLC7A11) and solute carrier family 2 member 1 (SLC2A1, also known as glucose transporter 1 (GLUT1)) in liver hepatocellular carcinoma (TCGA-LIHC). We found that SLC7A11 and SLC2A1 showed a moderate correlation in their expressions (Figure $3 C$ ), and that patients with higher expression of both SLC7A11 and SLC2A1 showed significantly lower survival rates than patients with low expression of SLC7A11 and/or SLC2A1 (Figure 3D-F, Supplementary Figure 2B-C). The synergistic negative effects between high expressions of SLC7A11 and SLC2A1 on outcomes were also observed in several other cancer types (Supplementary Figure 3). These results are consistent with the finding that co-targeting the L-cystine importer SLC7A11 and the glucose transporter SLC2A1 induces synthetic lethal cell death in glucose-deprived cell lines [58].

Third, to illustrate cSurvival's integrated workflow, and to show how cSurvival can bridge clinical and cell line studies, we assessed the Nrf2 (nuclear factor erythroid 2 related factor 2 (NFE2L2))Keap1 (Kelch-like erythroid cell-derived protein with CNC homology-associated protein 1 (KEAP1)) signalling pathway. Nrf2 is a master orchestrator of oxidative homeostasis and is primarily regulated by Keap1 [59]. In cancers, KEAP1 is frequently mutated, resulting in constitutively active Nrf2 that protects cancer cells from chemotherapeutic agents and facilitates cancer progression [59]. For 
example, Nrf2 is aberrantly activated in $\sim 30 \%$ of human lung cancers [60]. Using cSurvival, we found that expression or mutation of NFE2L2 and KEAP1 themselves showed no association with patient overall survival (Supplementary Figure 4). However, high expression of genes in the Nrf2-antioxidant response element (ARE) pathway (WikiPathways: WP4357) correlated strongly with poor prognosis in lung adenocarcinoma patients (Figure 4A-B). Consistent with this clinical finding, in DepMap's experimental genetic perturbation screens, KEAP1-mutated lung cancer cell lines were more sensitive to NFE2L2 knockout and knockdown (Figure 4C-F), consistent with KEAP1 mutation being the main driver for oncogenic Nrf2 activation [61,62]. Likewise, cell lines with higher expression of Nrf2-ARE pathway genes showed greater resistance to a potent oxidative stress inducer, menadione (BRDK78126613-001-28-5) (Figure 5A-B) [63]. Moreover, cSurvival analysis showed that the male A549 and the female $\mathrm{H} 2172$ cell lines both harbor deactivating KEAP1 mutations, manifest high Nrf2-ARE pathway expression, and show relatively high resistance to menadione (Figure 5). These findings are consistent with published reports that the A549 cell line is an excellent tool for studies on Nrf2 regulation and activity [64-66]; extending this, cSurvival analysis suggests that the female $\mathrm{H} 2172$ cell line is another excellent model to study Nrf2 function and can be used in conjunction with A549 to study sex-specific effects (Figure 5).

Together, these analyses demonstrate cSurvival's unique ability to support biomarker prognosis and interaction analysis via gene- and GS-level approaches, and to facilitate integrating clinical and experimental biomedical studies.

\section{DISCUSSION}

Cancer arises from accumulated genetic and epigenetic alterations, creating interactions that endow cancer cells with growth and survival advantages. Such functional relationships happen not only between genes, but also between GSs, or between genes and GSs. Correspondingly, regimens that combine multiple drugs targeting different genes/GSs have emerged as more effective and less toxic than mono-therapy approaches $[28,29]$. Pinpointing genetic interactions in cancers is thus an important research goal.

Here, we developed cSurvival, an open-source framework to identify potential genetic interactions and survey preclinical cell line tools. cSurvival offers innovative algorithms that can assess interactions between many types of cancer biomarkers, including GSs, as well as a curated database combining clinical and experimental data to generate synergistic biological insights. As shown by the three case studies, cSurvival sheds lights on genetic interactions in cancers and facilitates identification of preclinical cell line tools for mechanistic studies.

For long-term sustainability, we have automated data extraction from the $\mathrm{NCl}$ Genomic Data Commons (https://gdc.cancer.gov) [44] and will continuously follow consortium efforts, so that cSurvival uses up-to-date processing pipelines, human reference genome, and gene annotations. We also plan to expand the cSurvival database, incorporating resources such as the International Cancer Genome Consortium (ICGC) [67] and the Connectivity Map (CMap) [68,69]. Future iterations of cSurvival may also address the challenges of immune [70] and ethnicity [71] heterogeneities in human populations. 
Although we built cSurvival to analyze molecular biomarkers in cancers, its analytical approaches can also be applied to other types of (bio) markers and/or other diseases. For instance, its algorithms to search for optimal cutoffs for interaction analysis could also apply to data from biomedical imaging [72] or drug cocktail effect assessment [73], which sometimes involve combinations of continuous and/or categorical variables.

In summary, cSurvival offers a curated database and innovative analytical pipelines to examine cancer biomarkers at high resolution. It complements existing resources such as cBioPortal [9], and enhances mechanistic investigation of malignancy etiologies using clinical correlations. Its intuitive yet flexible web interface makes it a handy tool for experimental and clinical researchers alike. 


\begin{abstract}
AVAILABILITY
cSurvival (https://tau.cmmt.ubc.ca/cSurvival) is free and open to all users and has no login requirement. The source code for building cSurvival's framework and database is available at GitHub (https://github.com/easygsea/cSurvival.git).
\end{abstract}

\title{
SUPPLEMENTARY DATA
}

Supplementary figures are available at Briefings in Bioinformatics online.

\section{ACKNOWLEDGEMENT}

We thank Dr. P.W. Laird (Van Andel Institute, Grand Rapids, MI), T. Lichtenberg (University of Chicago, Chicago, IL), Dr. C.A. Maxwell (The University of British Columbia, Vancouver, BC), Dr. H. Shen (Van Andel Institute, Grand Rapids, MI), Dr. Z. Zhou (The Chinese University of Hong Kong, Shatin, HK), and Taubert lab members for critical comments on the manuscript. Figure 1 was created with BioRender.com, Toronto, Canada.

\section{FUNDING}

This work was funded by grant support from The Canadian Institutes of Health Research (CIHR; PJT153199); and the Natural Sciences and Engineering Research Council of Canada (NSERC; RGPIN2018-05133 to S.T., RGPIN-2017-04722 to X.Z.), and a Canada Research Chair (No. 950231363 to X.Z.). S.J.M.J. acknowledges funding from the Canada Research Chairs program. Funding for open access charges: Canadian Institutes of Health Research (CIHR; PJT-153199).

Xuanjin Cheng is a research associate in Medical Genetics at the University of British Columbia. Her research combines bioinformatics and molecular biology to study gene regulation in development and disease.

Yongxing Liu is a fourth-year Mathematics student at the University of British Columbia. He is interested in data science, machine learning, and bioinformatics.

Jiahe Wang is currently a Computer Science Master student at Simon Fraser University with a concentration on Big Data and Machine Learning.

Yujie Chen is an undergraduate student majoring in Statistics at the University of British Columbia, and her research interest is Machine Learning and Bioinformatics.

Andrew Gordon Robertson is an analyst with BC Cancer's Canada's Michael Smith Genome Sciences Centre in Vancouver Canada. He has contributed to many TCGA, PanCancer Atlas, and GDAN projects and publications. 
Xuekui Zhang is a Tier 2 Canada Research Chair in biostatistics and bioinformatics and an assistant professor in the Department of Mathematics and Statistics at the University of Victoria.

Steven J.M. Jones is co-director and head of bioinformatics at the Genome Sciences Centre, BC Cancer. He is also a Professor in Medical Genetics at the University of British Columbia.

Stefan Taubert is an associate professor in in Medical Genetics at the University of British Columbia who studies gene regulation in health and disease.

\section{CONFLICT OF INTEREST}

The authors declare no competing interests. 
bioRxiv preprint doi: https://doi.org/10.1101/2021.11.15.468756; this version posted November 19, 2021. The copyright holder for this preprint (which was not certified by peer review) is the author/funder. All rights reserved. No reuse allowed without permission.

Advanced cancer biomarker studies with cSurvival

\section{REFERENCES}

1. Kleinbaum DG, Klein M. Survival Analysis: A Self-Learning Text, Third Edition. 2012.

2. Schober $P$, Vetter TR. Survival Analysis and Interpretation of Time-to-Event Data: The Tortoise and the Hare. Anesth. Analg. 2018; 127:792-798

3. Györffy B, Lanczky A, Eklund AC, et al. An online survival analysis tool to rapidly assess the effect of 22,277 genes on breast cancer prognosis using microarray data of 1,809 patients. Breast Cancer Res. Treat. 2010; 123:725-731

4. Zheng $\mathrm{H}$, Zhang $\mathrm{G}$, Zhang $\mathrm{L}$, et al. Comprehensive Review of Web Servers and Bioinformatics Tools for Cancer Prognosis Analysis. Front. Oncol. 2020; 10

5. Tomczak K, Czerwińska P, Wiznerowicz M. The Cancer Genome Atlas (TCGA): an immeasurable source of knowledge. Contemp. Oncol. 2015; 19:A68-A77

6. Liu J, Lichtenberg T, Hoadley KA, et al. An Integrated TCGA Pan-Cancer Clinical Data Resource to Drive High-Quality Survival Outcome Analytics. Cell 2018; 173:400-416.e11

7. GenomeOC. Therapeutically Applicable Research to Generate Effective Treatments. Off. Cancer Genomics 2013;

8. Cerami1 E, Gao J, Dogrusoz U, et al. The cBio Cancer Genomics Portal: An Open Platform for Exploring Multidimensional Cancer Genomics Data. Cancer Discov. 2012; 2:401-404

9. Gao J, Aksoy BA, Dogrusoz U, et al. Integrative Analysis of Complex Cancer Genomics and Clinical Profiles Using the cBioPortal. Sci. Signal. 2013; 6:pl1

10. Zhang G, Wang Q, Yang M, et al. OSpaad: An online tool to perform survival analysis by integrating gene expression profiling and long-term follow-up data of 1319 pancreatic carcinoma patients. Mol. Carcinog. 2020; 59:304-310

11. Park S-J, Yoon B-H, Kim S-K, et al. GENT2: an updated gene expression database for normal and tumor tissues. BMC Med. Genomics 2019; 12:101

12. Goswami CP, Nakshatri H. PROGgeneV2: enhancements on the existing database. BMC Cancer 2014; $14: 970$

13. Aguirre-Gamboa R, Gomez-Rueda H, Martínez-Ledesma E, et al. SurvExpress: An Online Biomarker Validation Tool and Database for Cancer Gene Expression Data Using Survival Analysis. PLoS ONE 2013; 8:e74250

14. Mizuno H, Kitada K, Nakai K, et al. PrognoScan: a new database for meta-analysis of the prognostic value of genes. BMC Med. Genomics 2009; 2:18

15. Nagy Á, Munkácsy G, Győrffy B. Pancancer survival analysis of cancer hallmark genes. Sci. Rep. $2021 ; 11: 6047$

16. Liu C-J, Hu F-F, Xia M-X, et al. GSCALite: a web server for gene set cancer analysis.

Bioinformatics 2018; 34:3771-3772

17. Chandrashekar DS, Bashel B, Balasubramanya SAH, et al. UALCAN: A Portal for Facilitating Tumor Subgroup Gene Expression and Survival Analyses. Neoplasia N. Y. N 2017; 19:649-658 18. Tang Z, Kang B, Li C, et al. GEPIA2: an enhanced web server for large-scale expression profiling and interactive analysis. Nucleic Acids Res. 2019; 47:W556-W560

19. Han S, Kim D, Kim Y, et al. CAS-viewer: web-based tool for splicing-guided integrative analysis of multi-omics cancer data. BMC Med. Genomics 2018; 11:25

20. Anaya J. OncoLnc: linking TCGA survival data to mRNAs, miRNAs, and IncRNAs. PeerJ Comput. Sci. 2016; 2:e67

21. Goldman MJ, Craft B, Hastie M, et al. Visualizing and interpreting cancer genomics data via the Xena platform. Nat. Biotechnol. 2020; 38:675-678

22. Zhang J, Baran J, Cros A, et al. International Cancer Genome Consortium Data Portal-a onestop shop for cancer genomics data. Database J. Biol. Databases Curation 2011; 2011:bar026 23. Gentles AJ, Newman AM, Liu CL, et al. The prognostic landscape of genes and infiltrating immune cells across human cancers. Nat. Med. 2015; 21:938-945

24. Bradburn MJ, Clark TG, Love SB, et al. Survival Analysis Part III: Multivariate data analysis choosing a model and assessing its adequacy and fit. Br. J. Cancer 2003; 89:605-611

25. Ma X, Huang R, Wu X, et al. Dualmarker: a flexible toolset for exploratory analysis of combinatorial dual biomarkers for clinical efficacy. BMC Bioinformatics 2021; 22:127

26. Magen A, Sahu AD, Lee JS, et al. Beyond Synthetic Lethality: Charting the Landscape of Pairwise Gene Expression States Associated with Survival in Cancer. Cell Rep. 2019; 28:938-948.e6

27. Yu J, Zhou D, Yang X, et al. TRIB3-EGFR interaction promotes lung cancer progression and defines a therapeutic target. Nat. Commun. 2020; 11:3660

28. Mokhtari RB, Homayouni TS, Baluch N, et al. Combination therapy in combating cancer.

Oncotarget 2017; 8:38022-38043

29. Ledford H. Cocktails for cancer with a measure of immunotherapy. Nature 2016; 532:162-164 
bioRxiv preprint doi: https://doi.org/10.1101/2021.11.15.468756; this version posted November 19, 2021. The copyright holder for this preprint (which was not certified by peer review) is the author/funder. All rights reserved. No reuse allowed without permission.

Advanced cancer biomarker studies with cSurvival

30. Zhang J, Zhang S. Discovery of cancer common and specific driver gene sets. Nucleic Acids Res. 2017; 45:e86

31. Subramanian A, Tamayo P, Mootha VK, et al. Gene set enrichment analysis: A knowledge-based approach for interpreting genome-wide expression profiles. Proc. Natl. Acad. Sci. U. S. A. 2005; 102:15545-15550

32. Maleki F, Ovens K, Hogan DJ, et al. Gene Set Analysis: Challenges, Opportunities, and Future Research. Front. Genet. 2020; 11:654

33. Zheng $\mathrm{X}, \mathrm{Amos} \mathrm{Cl}$, Frost HR. Comparison of pathway and gene-level models for cancer prognosis prediction. BMC Bioinformatics 2020; 21:76

34. Rehman SK, Haynes J, Collignon E, et al. Colorectal Cancer Cells Enter a Diapause-like DTP State to Survive Chemotherapy. Cell 2021; 184:226-242.e21

35. Goeman JJ, Oosting J, Cleton-Jansen A-M, et al. Testing association of a pathway with survival using gene expression data. Bioinformatics 2005; 21:1950-1957

36. Tsherniak A, Vazquez F, Montgomery PG, et al. Defining a Cancer Dependency Map. Cell 2017; 170:564-576.e16

37. Meyers RM, Bryan JG, McFarland JM, et al. Computational correction of copy-number effect improves specificity of CRISPR-Cas9 essentiality screens in cancer cells. Nat. Genet. 2017; 49:17791784

38. Dempster JM, Rossen J, Kazachkova M, et al. Extracting Biological Insights from the Project Achilles Genome-Scale CRISPR Screens in Cancer Cell Lines. bioRxiv 2019; 720243

39. Ghandi M, Huang FW, Jané-Valbuena J, et al. Next-generation characterization of the Cancer Cell Line Encyclopedia. Nature 2019; 569:503-508

40. Cheng X, Yan J, Liu Y, et al. eVITTA: a web-based visualization and inference toolbox for transcriptome analysis. Nucleic Acids Res. 2021; 49:W207-W215

41. Hoadley KA, Yau C, Hinoue T, et al. Cell-of-Origin Patterns Dominate the Molecular Classification of 10,000 Tumors from 33 Types of Cancer. Cell 2018; 173:291-304.e6

42. Ellrott K, Bailey MH, Saksena G, et al. Scalable Open Science Approach for Mutation Calling of Tumor Exomes Using Multiple Genomic Pipelines. Cell Syst. 2018; 6:271-281.e7

43. Zhou W, Laird PW, Shen H. Comprehensive characterization, annotation and innovative use of Infinium DNA methylation BeadChip probes. Nucleic Acids Res. 2017; 45:e22

44. Heath AP, Ferretti V, Agrawal S, et al. The NCl Genomic Data Commons. Nat. Genet. 2021; 53:257-262

45. Colaprico A, Silva TC, Olsen C, et al. TCGAbiolinks: an R/Bioconductor package for integrative analysis of TCGA data. Nucleic Acids Res. 2016; 44:e71

46. Silva TC, Colaprico A, Olsen C, et al. TCGA Workflow: Analyze cancer genomics and epigenomics data using Bioconductor packages. F1000Research 2016; 5:1542

47. Mounir M, Lucchetta M, Silva TC, et al. New functionalities in the TCGAbiolinks package for the study and integration of cancer data from GDC and GTEx. PLoS Comput. Biol. 2019; 15:e1006701

48. Carlson M. org.Hs.eg.db: Genome wide annotation for Human. R package version 3.11.4. 2020;

49. Corsello SM, Nagari RT, Spangler RD, et al. Discovering the anti-cancer potential of non-oncology drugs by systematic viability profiling. Nat. Cancer 2020; 1:235-248

50. Nusinow DP, Szpyt J, Ghandi M, et al. Quantitative Proteomics of the Cancer Cell Line Encyclopedia. Cell 2020; 180:387-402.e16

51. Corsello SM, Bittker JA, Liu Z, et al. The Drug Repurposing Hub: a next-generation drug library and information resource. Nat. Med. 2017; 23:405-408

52. Therneau TM, Grambsch PM. Modeling Survival Data: Extending the Cox Model. 2000;

53. Lausen B, Schumacher M. Maximally Selected Rank Statistics. Biometrics 1992; 48:73-85

54. Hilsenbeck SG, Clark GM. Practical p-value adjustment for optimally selected cutpoints. Stat.

Med. 1996; 15:103-112

55. R Core Team. R: A language and environment for statistical computing. R Foundation for

Statistical Computing, Vienna, Austria. URL https://www.R-project.org/. 2020;

56. Kassambara A, Kosinski M, Biecek P. survminer: Drawing Survival Curves using 'ggplot2'. R package version 0.4.9. https://rpkgs. datanovia.com/survminer/index.html. 2021;

57. Wickham H, Averick M, Bryan J, et al. Welcome to the Tidyverse. J. Open Source Softw. 2019; 4:1686

58. Joly JH, Delfarah A, Phung PS, et al. A synthetic lethal drug combination mimics glucose deprivation-induced cancer cell death in the presence of glucose. J. Biol. Chem. 2020; 295:13501365

59. Jaramillo MC, Zhang DD. The emerging role of the Nrf2-Keap1 signaling pathway in cancer.

Genes Dev. 2013; 27:2179-2191 
bioRxiv preprint doi: https://doi.org/10.1101/2021.11.15.468756; this version posted November 19, 2021. The copyright holder for this preprint (which was not certified by peer review) is the author/funder. All rights reserved. No reuse allowed without permission.

Advanced cancer biomarker studies with cSurvival

60. Collisson EA, Campbell JD, Brooks AN, et al. Comprehensive molecular profiling of lung adenocarcinoma. Nature 2014; 511:543-550

61. de la Vega MR, Chapman E, Zhang DD. NRF2 and the hallmarks of cancer. Cancer Cell 2018; 34:21-43

62. Singh A, Misra V, Thimmulappa RK, et al. Dysfunctional KEAP1-NRF2 Interaction in Non-SmallCell Lung Cancer. PLoS Med. 2006; 3:e420

63. Thor H, Smith MT, Hartzell P, et al. The metabolism of menadione (2-methyl-1,4-naphthoquinone) by isolated hepatocytes. A study of the implications of oxidative stress in intact cells. J. Biol. Chem. 1982; 257:12419-12425

64. Ohta T, lijima K, Miyamoto M, et al. Loss of Keap1 Function Activates Nrf2 and Provides

Advantages for Lung Cancer Cell Growth. Cancer Res. 2008; 68:1303-1309

65. Wang X-J, Sun Z, Villeneuve NF, et al. Nrf2 enhances resistance of cancer cells to chemotherapeutic drugs, the dark side of Nrf2. Carcinogenesis 2008; 29:1235-1243

66. Gong M, Li Y, Ye X, et al. Loss-of-function mutations in KEAP1 drive lung cancer progression via KEAP1/NRF2 pathway activation. Cell Commun. Signal. CCS 2020; 18:98

67. . International network of cancer genome projects. Nature 2010; 464:993-998

68. Subramanian A, Narayan R, Corsello SM, et al. A Next Generation Connectivity Map: L1000

Platform and the First 1,000,000 Profiles. Cell 2017; 171:1437-1452.e17

69. Lamb J, Crawford ED, Peck D, et al. The Connectivity Map: Using Gene-Expression Signatures to Connect Small Molecules, Genes, and Disease. Science 2006; 313:1929-1935

70. Thorsson V, Gibbs DL, Brown SD, et al. The Immune Landscape of Cancer. Immunity 2018; 48:812-830.e14

71. Yuan J, Hu Z, Mahal BA, et al. Integrated Analysis of Genetic Ancestry and Genomic Alterations across Cancers. Cancer Cell 2018; 34:549-560.e9

72. Wang JW, Williams M. Repurposing Routine Imaging for Cancer Biomarker Discovery Using Machine Learning. Handb. Artif. Intell. Healthc. Vol 1 - Adv. Appl. 2022; 153-176

73. Gibbons JA, de Vries M, Krauwinkel W, et al. Pharmacokinetic Drug Interaction Studies with Enzalutamide. Clin. Pharmacokinet. 2015; 54:1057-1069

74. Gu Z, Gu L, Eils R, et al. circlize implements and enhances circular visualization in R.

Bioinformatics 2014; 30:2811-2812 


\section{FIGURE LEGENDS}

Figure 1. Overview of the cSurvival analytical framework. TCGA, The Cancer Genome Atlas; TARGET, Therapeutically Applicable Research to Generate Effective Treatments; DepMap, Dependency Map; GS, gene set; eVITTA, easy Visualization and Inference Toolbox for Transcriptome Analysis; Cat., categorical predictor; Cont., continuous predictor; Mut, mutated; HR, hazard ratio; $P$, $P$-value; $P$.adj, adjusted $P$-value; Cor, correlation coefficient.

Figure 2. Overview of the cSurvival database. (A) The circos plot (rendered with circulize [74]) shows the distribution of tumor and cell line datasets: Inner to outer: example outputs; project names; histograms showing total number of cases per study; box plots showing distribution of survival days (TCGA, TARGET) or dependency scores/cell viabilities (DepMap), numbers denoting 3-, 5-, and 10year survival rates from inner to outer; study names. (B) The histogram shows the distribution of GS libraries from eVITTA; numbers denote the number of GSs per library. TCGA, The Cancer Genome Atlas; TARGET, Therapeutically Applicable Research to Generate Effective Treatments; DepMap, Dependency Map; GS, gene set; eVITTA, easy Visualization and Inference Toolbox for Transcriptome Analysis.

Figure 3. Evaluation study on autophagy-dependent survival in colorectal cancers and synergistic effects between high expression of SLC7A11 and SLC2A1 in liver cancers. The survival curves (A) and forest plot (B), censored at 10 years, show correlation between GO autophagy signature (GO: 0010506) and overall survival in colorectal cancers (TCGA-COAD and READ) $(P=0.012, P$.adj $=0.03, \mathrm{KM}$ log-rank; HR $=1.7, P=0.017, P$.adj $=0.05$, Cox PH likelihood ratio). The scatter plot (C) shows a moderate correlation between expression of SLC7A11 and SLC2A1 (Pearson's correlation coefficient $=0.35, P=3.2 \mathrm{e}-12$ ). The survival curves (D), censored at 10 years, show significant differences in overall survival rates among patients with liver hepatocellular carcinoma (TCGA-LIHC) stratified by SLC7A11 and SLC2A1 expression levels $(P=1 \mathrm{e}-07, P$.adj < $0.01, \mathrm{KM}$ log-rank; $P=1.7 \mathrm{e}-06, P$.adj $<0.01$, Cox $\mathrm{PH}$ likelihood ratio). The survival curves (E) and forest plots $(\mathbf{F})$, censored at 10 years, show a lower overall survival rate in $\mathrm{LIHC}$ patients with high expression of both SLC7A11 and SLC2A1 than in patients with low expression of SLC7A11 and/or SLC2A1 $(P=4.2 \mathrm{e}-08, P$.adj < 0.01, KM log-rank; HR = 3.38, $P=4.9 \mathrm{e}-06, P$.adj < 0.01, Cox PH likelihood ratio). GO, gene ontology; TCGA, The Cancer Genome Atlas; COAD, colon adenocarcinoma; READ, rectum adenocarcinoma; $P, P$-value; $P$.adj, adjusted $P$-value; KM, KaplanMeier; PH, proportional-hazard; HR, hazard ratio; LIHC, liver hepatocellular carcinoma.

Figure 4. High expression of the Nrf2-ARE pathway correlates with poor prognosis and KEAP1 mutation in lung cancers. The survival curves (A) and forest plot (B), censored at 10 years, show correlation between high expression of the Nrf2-ARE pathway and poor prognosis in TCGA-LUAD cases $(P=0.00015, P$.adj $<0.01, \mathrm{KM}$ log-rank; HR $=1.84, P=0.00027, P$.adj $<0.01, \mathrm{Cox} \mathrm{PH}$ likelihood ratio). Shades reflect $95 \%$ confidence intervals in survival curves in (A). The density and box plots show lung cancer cell lines with an inactivating KEAP1 mutation (red) being more sensitive 
to NFE2L2 CRISPR-Cas9 knockout ( $P=4.1 \mathrm{e}-07$, two-tailed Wilcoxon test) (C, D) and RNAi knockdown ( $P=8.4 \mathrm{e}-05$, two-tailed Wilcoxon test) (E, F). ARE, antioxidant response element; TCGA, The Cancer Genome Atlas; LUAD, lung adenocarcinoma; $P$, $P$-value; $P$.adj, adjusted $P$-value; KM, Kaplan-Meier; HR, hazard ratio; PH, proportional-hazard; RNAi, RNA interference; Chronos, an algorithm for inferring gene knockout fitness effects; DEMETER2, gene dependency estimates for RNAi datasets.

Figure 5. The male A549 and the female H2172 cell lines are top candidates for studies on Nrf2 regulation and activity. The density $(\mathbf{A})$ and box $(\mathbf{B})$ plots show that lung cancer cell lines with higher expression levels of Nrf2-ARE pathway genes exhibit higher resistance to the oxidative stress inducer menadione (BRD-K78126613-001-28-5, $P=0.00014, P$.adj < 0.01, two-tailed Wilcoxon test). The density (C) and box (D) plots show that lung cancer cell lines with different KEAP1 mutation status and expression levels of Nrf2-ARE pathway genes show sensitivity differences to NFE2L2 knockout ( $P=2.6 \mathrm{e}-06, P$.adj $<0.01$, Kruskal-Wallis rank sum test). The A549 and the $\mathrm{H} 2172$ cell lines are highlighted in triangle shape with darker color and labelled in the box plots. ARE, antioxidant response element; $P, P$-value; $P$.adj, adjusted $P$-value; Chronos, an algorithm for inferring gene knockout fitness effects. 
bioRxiv preprint doi: https://doi.org/10.1101/2021.11.15.468756; this version posted November 19, 2021. The copyright holder for this

preprint (which was not certified by peer review) is the author/funder. All rights reserved. No reuse allowed without permission.

Figure 1

Source data

Extraction, wrangling, curation, gene ID mapping \& format transformation

TCGA

10,973 adult tumors

33 cancer types

\section{TARGET}

4,995 pediatric tumors

7 cancer types

\section{DepMap}

1,747 cancer cell lines

17,393 CRISPR \& 17,309 RNAi perturbations 4,686 compounds

\section{cSurvival curated database}

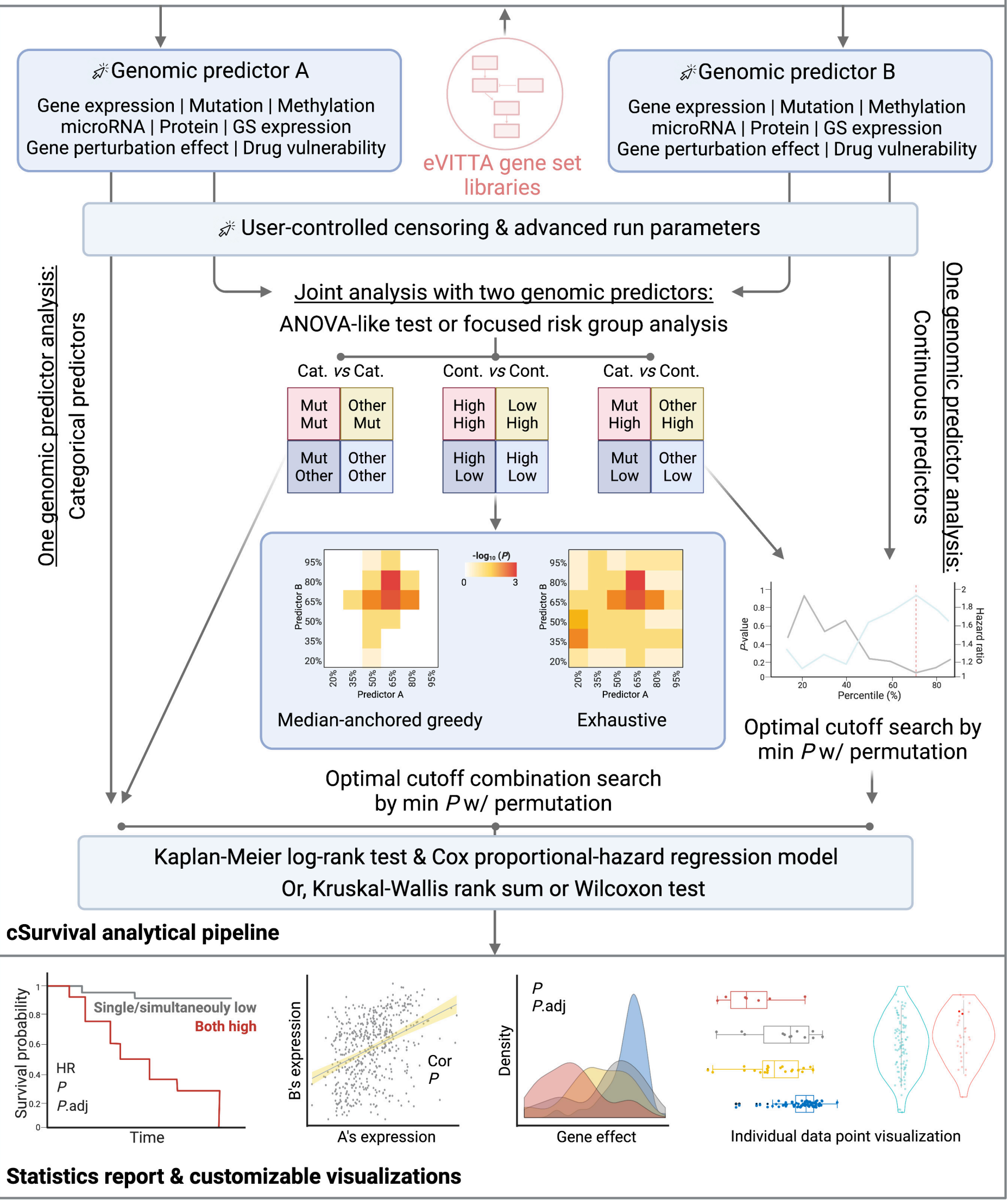


Figure 2

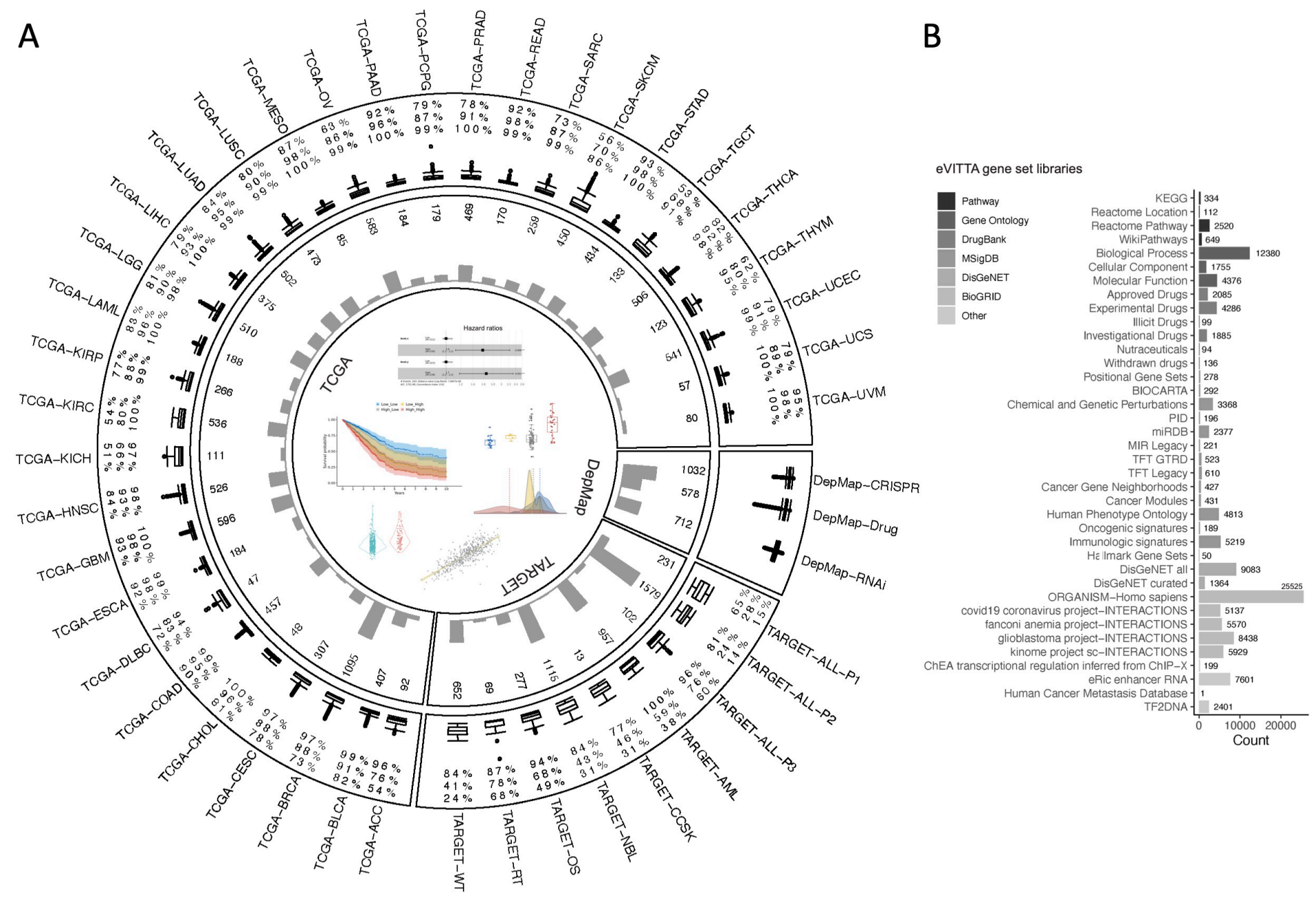


Figure 3

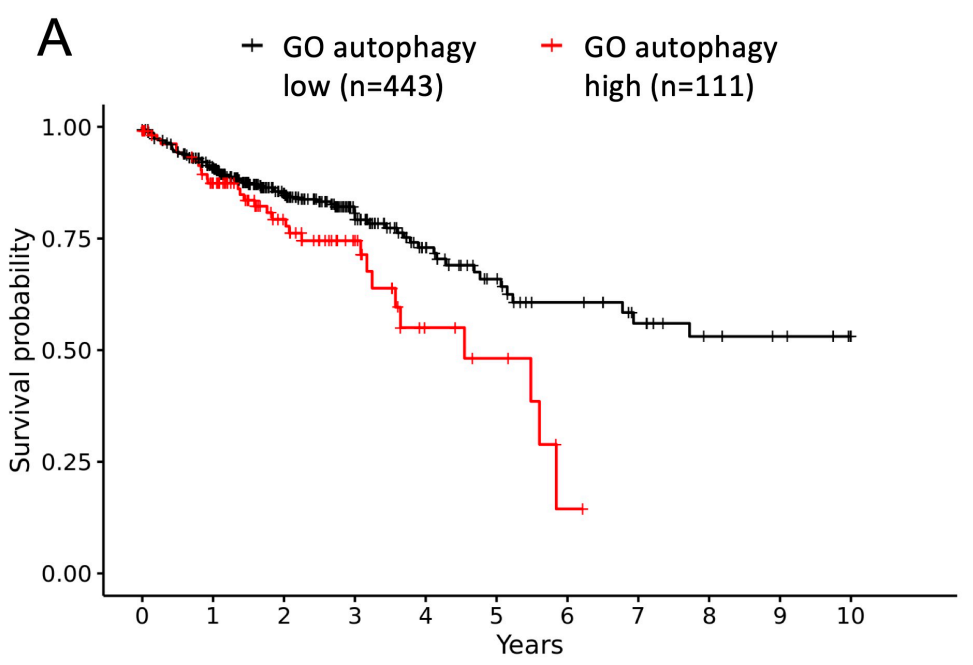

B

Hazard ratio

level $\quad \stackrel{\operatorname{Low}}{(N=443)}$

$\underset{\substack{H i g h \\(N=111)}}{\ln }$

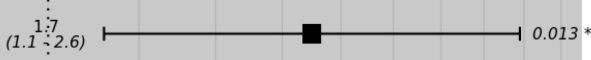
\# Events: 112; Global p-value (Log-Rank): 0.016993
$\begin{array}{llllllllll}\text { AIC: } 1227.39 ; \text { Concordance Index: } 0.53 & 1.2 & 1.4 & 1.6 & 1.8 & 2 & 2.2 & 2.4 & 2.6 & 2.8\end{array}$

\section{C}

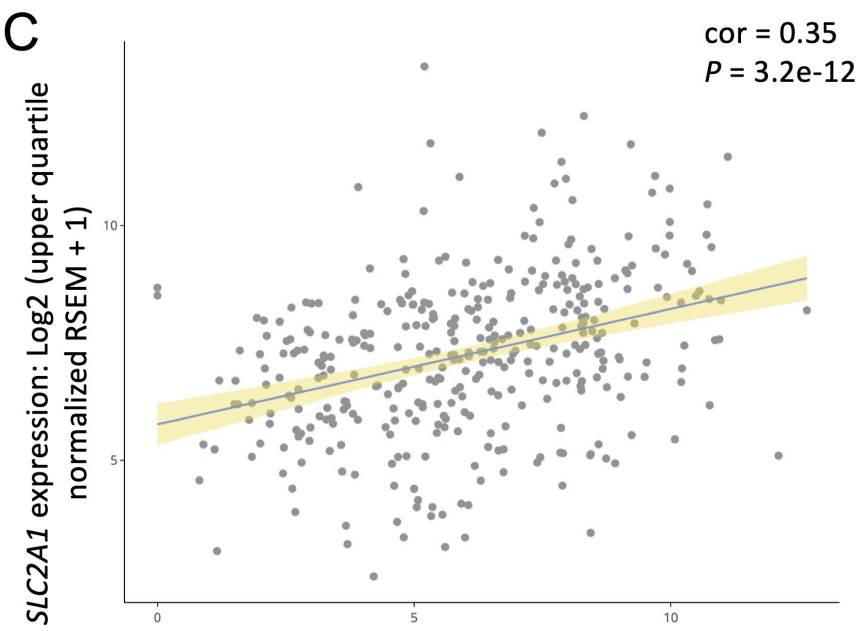

SLC7A11 expression: Log2 (upper quartile normalized RSEM + 1)

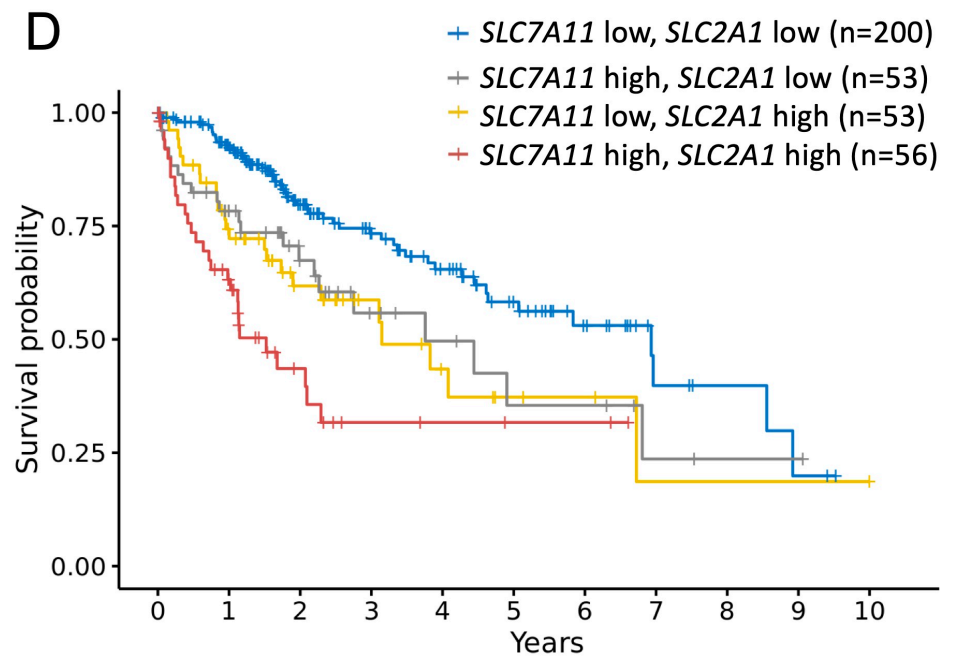

E

+ SLC7A11 and/or SLC2A1 low ( $\mathrm{n}=320)$

+ SLC7A11 and SLC2A1 both high $(\mathrm{n}=42)$

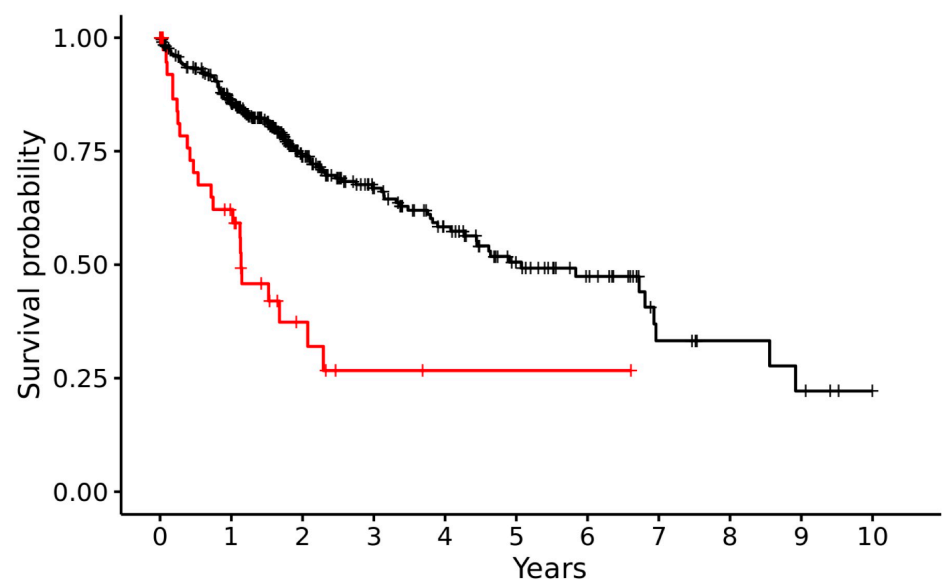

F

\section{Hazard ratio}

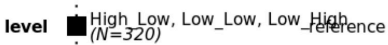

$\underset{(N=42)}{\text { High High }}$

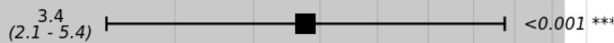

\# Eventś: 127; Global p-value (Log-Rank): 4.9394e-06 $2503.5 \quad 4 \quad 4.5 \quad 5 \quad 5.566 .5$ AIC: 1276.17; Concordance Index: 0.58 
Figure 4
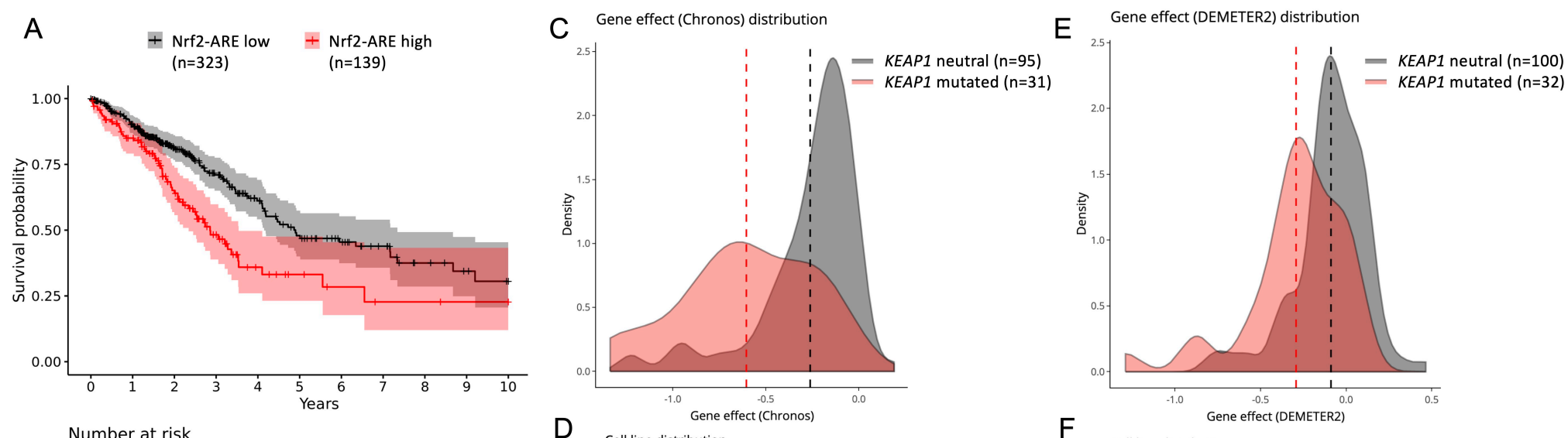

Number at risk

\section{Cell line distribution}

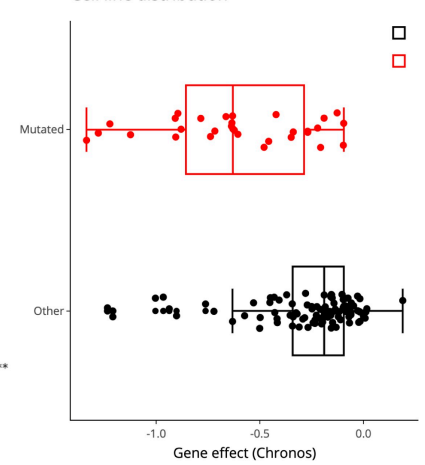

F

KEAP1 neutral $(\mathrm{n}=95)$ KEAP1 mutated $(n=31)$

Cumulative number of events

$$
\begin{array}{llllllllllll}
\multicolumn{1}{c}{\text { Cumulative number of events }} \\
\mathbf{-} & 0 & 29 & 51 & 67 & 79 & 92 & 94 & 95 & 98 & 99 & 100 \\
- & 1 & 20 & 41 & 54 & 60 & 61 & 62 & 63 & 63 & 63 & 63
\end{array}
$$

B

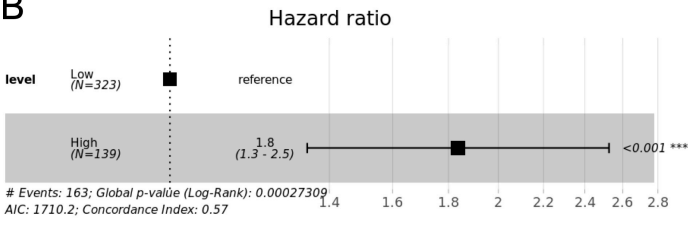

Hazard ratio

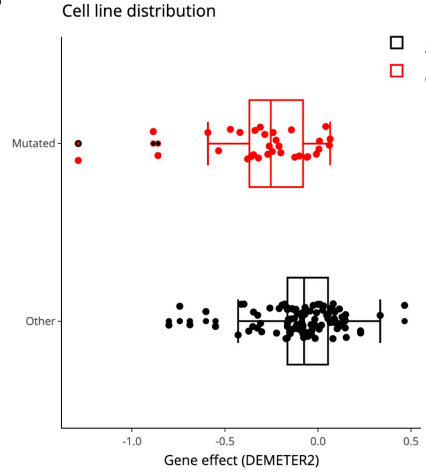




\section{Figure 5}

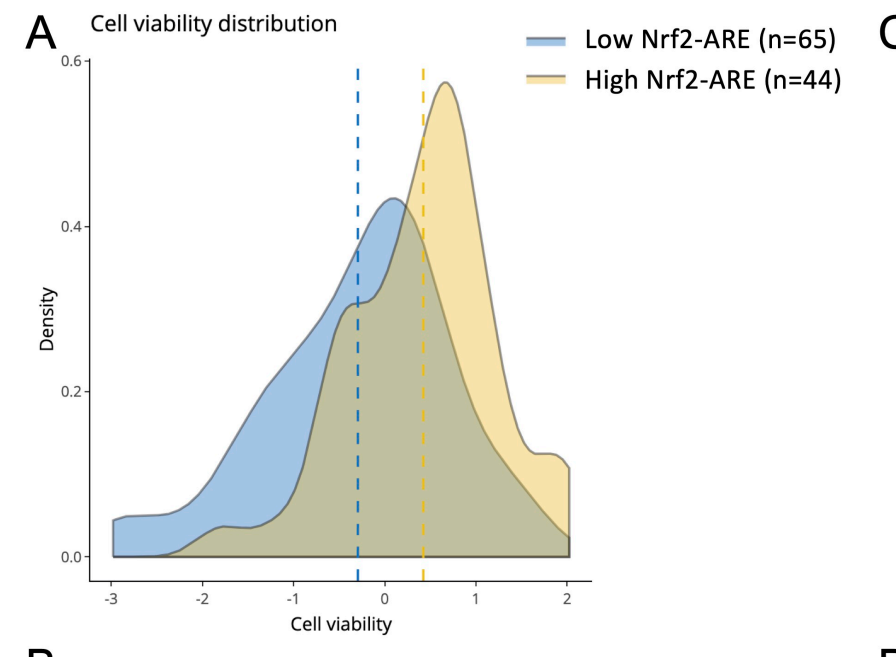

C Gene effect (Chronos) distribution

B Cell line distribution

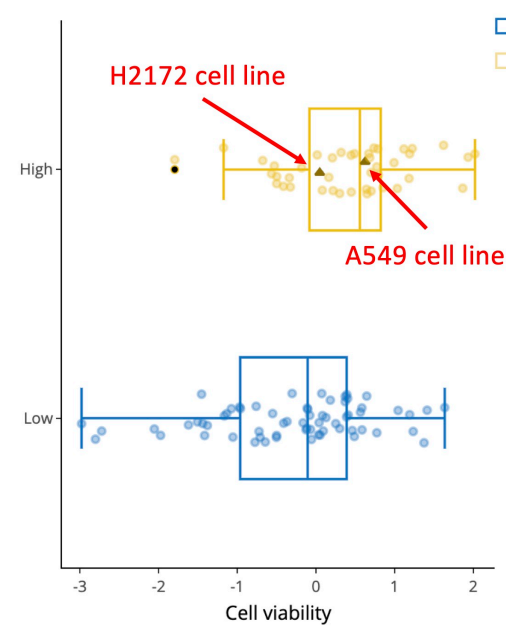

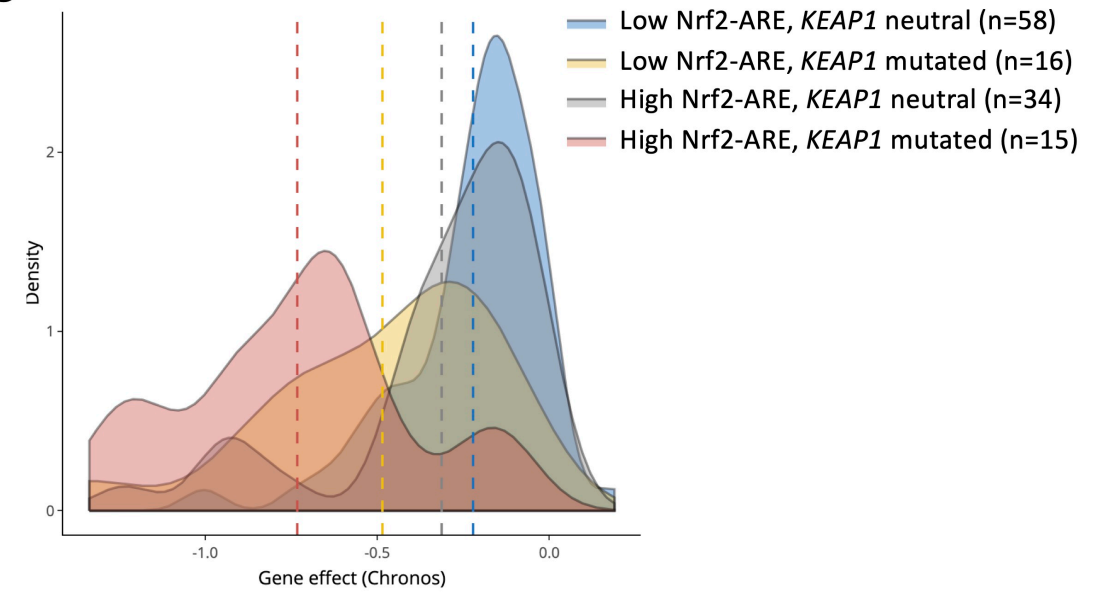

D Cell line distribution

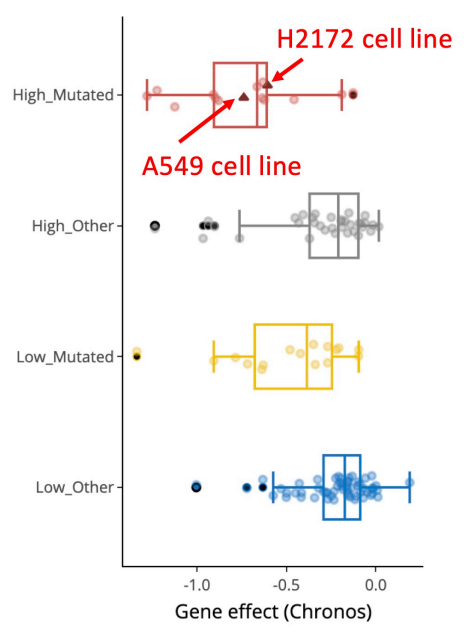

$\square \quad$ Low Nrf2-ARE, KEAP1 neutral $(\mathrm{n}=58)$

$\square$ Low Nrf2-ARE, KEAP1 mutated $(n=16)$

$\square$ High Nrf2-ARE, KEAP1 neutral $(n=34)$

$\square$ High Nrf2-ARE, KEAP1 mutated $(n=15)$
Low Nrf2-ARE $(n=65)$

High Nrf2-ARE $(n=44)$ 